\title{
PA-069 CYP2B6 GENOTYPE BASED EFAVIRENZ DOSE RECOMMENDATIONS DURING RIFAMPICIN-BASED ANTITUBERCULOSIS CO-TREATMENT FOR A SUB-SAHARAN AFRICA POPULATION
}

Jackson Mukonzo, ${ }^{1}$ Ronald Kuteesa, ${ }^{1}$ Jasper Ogwal-Okeng, ${ }^{1}$ Lars L. Gustafsson, ${ }^{2}$ Joel Owen, ${ }^{3}$ Eleni Aklillu'. 'Makerere University, Uganda; ${ }^{2} \mathrm{KI}$, Sweden; ${ }^{3}$ Union University, United States of America

\subsection{6/bmjgh-2016-000260.102}

Background Pharmacogenetics is a major determinant of the EFV-rifampicin interaction during HIV-TB co-treatment. We assessed genetic factors that influence EFV PK, treatment outcomes and provide genotype-based EFV dose recommendations for adult TB-HIV-1 co-infected Ugandans receiving rifampicin based anti-tuberculosis co-treatment.

Methods Steady state plasma EFV concentrations $(n=1216)$ from 158 HIV-TB co-infected patients (76 females) treated with efavirenz/lamivudine/zidovidine and rifampicin-based TB treatment were analysed. Patient genotypes for CYP2B6 (*6 \& *11), CYP3A5 ("3,*6 \& *7) and ABCB1c.4046A>G, baseline biochemistries and CD4 and viral load change from baseline were determined. A one-compartment population PK model with firstorder absorption (NONMEMTM) was used to estimate genotype effects on EFV PK. Population genotype-frequency-based PK simulations predicted AUCs and trough concentrations were compared between the product label / known reference values and different dose simulations.

Results Compared to CYP2B6*1/*1, EFV post-induction CL/F was 2.5 and 1.7 times higher in CYP2B6*6/*6 and CYP2B6*1*/ 6 , respectively. A $23 \%$ increase in F1 was observed for the variant $\mathrm{ABCB} 1$ c. $4046 \mathrm{~A}>\mathrm{G}$. EFV mean AUC was significantly higher in CYP2B6"6/*6 genotypes compared to CYP2B6"1/*1 $(\mathrm{p}<0.0001)$. Simulated AUCs for a $600 \mathrm{mg}$ EFV dose were 1.2 and 2.4 times greater than the product label mean AUC for the Ugandan population in general and CYP $2 B 6 * 6 / * 6$ genotypes, respectively. EFV daily doses of $450 \mathrm{mg}$ and $250 \mathrm{mg}$ for the general population and $C Y P 2 B 6 * 6 / * 6$ genotypes respectively yielded simulated exposures that were comparable to the product label. Overall, only $8.9 \%$ patients had HIV RNA $>40$ copies $/ \mathrm{mL}$ after 84 days of treatment.

Conclusions During rifampicin co-treatment, daily doses of $450 \mathrm{mg}$ and $250 \mathrm{mg}$ might meet the EFV dosing needs of HIV-TB infected Ugandans in general and CYP2B6*6 homozygous variants, respectively. 